\title{
VARIABLES PREDICTORAS DE LOS NIVELES DE SEDENTARISMO EN POBLACIÓN DE 18 A 60 AÑOS, MEDELLÍN 2012
}

\section{PREDICTOR VARIABLES SEDENTARISM LEVELS IN POPULATION 18 TO 60 YEARS, MEDELLIN 2012}

\author{
José Armando Vidarte ${ }^{1}$, Consuelo Vélez ${ }^{2}$, Lisette Ethel Iglesias ${ }^{3}$, Yazmín Galvis ${ }^{4}$
}

\begin{abstract}
${ }^{1}$ Ph.D. en Actividad Física y Deporte, Universidad Autónoma de Manizales, Antigua Estación del ferrocarril, Manizales, Caldas, Colombia, e-mail: jovida@autonoma.edu.co; ${ }^{2}$ Ph.D. en Salud Pública, Universidad Autónoma de Manizales, Antigua estación del Ferrocarril, Manizales, e-mail: cva@autonoma.edu.co; ${ }^{3}$ Educadora Física, Fisioterapeuta, Candidata a Magíster en Intervención Integral en el deportista, e- mail: lisetteiglesias@hotmail.com; ${ }^{4}$ Fisioterapeuta, Candidata a Magíster en Intervención Integral en el deportista, e-mail: yazgalvis@hotmail.com. Dirección para Correspondencia: Universidad Autónoma de Manizales, Antigua estación del Ferrocarril, Manizales, e-mail: jovida@autonoma.edu.co
\end{abstract}

Rev. U.D.C.A Act. \& Div. Cient. 17(1): 65-72, Enero-Junio, 2014

\section{RESUMEN}

El sedentarismo es un factor asociado con la morbilidad y la mortalidad de enfermedades crónicas y degenerativas, como la diabetes y los problemas cardiovasculares, entre otros. El objetivo de esta investigación fue determinar las variables que predicen los niveles de sedentarismo en la población entre 18 y 60 años, de la ciudad de Medellín, en el 2012. Se realizó un estudio descriptivo transversal, correlacional, con la participación de 529 personas. Para la recolección de la información, se utilizó un instrumento con preguntas estructuradas y semiestructuradas, con el cual, se buscó dar cuenta de las variables sociodemográficas y para la definición del nivel de sedentarismo, se aplicó un test de medición, mediante una prueba física. Los resultados muestran cómo la prevalencia de sedentarismo fue de 76,2\% IC95\% (72,6\%; 78,8\%), las variables que predicen el sedentarismo en la población estudiada son el género, el índice de masa corporal (IMC) y el consumo de café, el modelo pronostica el $76,2 \%$ de los casos.

Palabras clave: Obesidad, prevalencia, actividad física, estilo de vida sedentario.

\section{SUMMARY}

Physical inactivity is a factor associated with morbidity and mortality of chronic and degenerative diseases like diabetes and cardiovascular problems, among others. The objective of this research was to determine the variables that predict levels of sedentary population between 18 and 60 years of the city of Medellin in 2012. A transversal descriptive, corre- lational study was conducted, with 529 people participating. For the collection of information an instrument with semistructured questions was used, which sought to give account of the socio-demographic variables and for the definition of physical inactivity level a measurement test was applied using a physical trial. The results show that the prevalence of physical inactivity was $76.2 \%$ IC95\% (72.6\%, $78.8 \%)$. The variables that predict of physical inactivity in the population studied are gender, body mass index (BMI) and coffee consumption; the model predicts $76.2 \%$ of cases

Key words: Obesity, prevalence, motor activity, sedentary lifestyle.

\section{INTRODUCCIÓN}

La Organización Mundial de la Salud (OMS, 2002) define el sedentarismo como "la poca agitación o movimiento" y establece que las personas sedentarias en sus actividades cotidianas son aquellas que no aumentan más del $10 \%$ la energía que gastan en el reposo, metabolismo basal o, como lo afirma Pate et al. (2008), se ha recurrido simplemente a considerar físicamente inactivos a quienes no alcanzan los niveles recomendados de actividad física moderada o intensa, es decir, se ha criticado la conducta sedentaria sin medirla directamente.

El panorama mundial muestra una permanente preocupación por este tema, teniendo claras las consecuencias que se generan por el sedentarismo en la población, situación que amerita una mirada más profunda y de preocupación por 
parte de los entes gubernamentales y estatales, ya que las cifras de inactividad física en la población cada día son mayores y los procesos que se desarrollan para su prevención, pareciera no alcanzan los resultados esperados.

Existe una relación causal entre el sedentarismo y las enfermedades graves, como la hipertensión arterial, el infarto agudo al miocardio, la diabetes, los eventos cerebro vasculares, la osteoporosis, el cáncer, enfermedades mentales, el sobrepeso, entre otras (Ramírez et al. 2004; Bouchard et al. 1994; Seron et al. 2010; Elizondo-Armendariz et al. 2005; McGinnis \& Foege, 1993; OMS, 2009).

En España, se han realizado estudios que han indagado sobre la prevalencia de actividad física en diferentes poblaciones, analizando la participación en ésta, pero con un nivel necesario para mejorar la salud. Blasco et al. (1996) y Capdevila et al. (2006) concluyen que la inactividad física de la población es un grave problema de salud entre los estudiantes universitarios y que son necesarias intervenciones encaminadas a la realización de mayor cantidad de actividad física.

La Organización Panamericana de la Salud, OPS (2005) afirma que se registraron aproximadamente 170.000 muertes en América Latina, a causa del sedentarismo. Lo anterior, se complementa con lo expuesto por el estudio argentino sobre sedentarismo, que pone de manifiesto cómo la tasa de mortalidad de las personas, que tienen niveles de actividad física altos y moderados, es menor que la de las personas que no realizan ninguna actividad física (Secretaría de Turismo, 2000).

En Chile, el problema con el sedentarismo muestra un porcentaje de personas que sí realizan actividad física muy bajo y el total de la población sedentaria es del 89,4\% (Serón et al. 2010). En Costa Rica, la principal causa de muerte son las enfermedades cardiovasculares y cada vez la situación es peor, porque se presenta en personas sedentarias, lo que pone en manifiesto la falta de medidas de prevención, ya que los niveles de obesidad están en gran parte de la población (Alemán \& Salazar, 2004).

En Colombia, diversos estudios muestran cómo el nivel de aptitud física de las personas presentan un indicador del $29 \%$ con obesidad y del $48 \%$, por encima del porcentaje de normalidad, en cuanto a grasa se refiere. Las personas cada vez son menos activas y no utilizan la cantidad de energía que obtienen de los alimentos que consumen diariamente, lo que lleva al aumento del peso corporal (García \& Correa, 2007; Martínez et al. 2008; Varela et al. 2011).

En Manizales, se estableció que la prevalencia de sedentarismo fue de $72,2 \%$, siendo mayor en las mujeres, con un $84 \%$ y variables, como la edad, el género, la frecuencia semanal de práctica, el perímetro cadera y la frecuencia cardiaca inicial explicaron el comportamiento de los niveles de sedentarismo, de manera significativa (Vidarte et al. 2012).

El objetivo fue determinar las variables que predicen los niveles de sedentarismo en población entre 18 y 60 años, de la ciudad de Medellín, para, de esta manera, a partir de sus resultados, contar con evidencia científica, que posibiliten a las autoridades municipales la creación y el fortalecimiento de políticas públicas, con miras a aportar elementos que, a futuro, puedan impactar sobre los perfiles epidemiológicos, con especial énfasis en las enfermedades crónicas y degenerativas.

\section{MATERIALES Y MÉTODOS}

Se realizó un estudio descriptivo transversal, con una fase correlacional.

La población evaluada fueron personas con edades entre 18 y 60 años de la ciudad de Medellín. Para el cálculo de la muestra, se realizó el siguiente procedimiento: utilizando la prevalencia del sedentarismo establecido en la ciudad de Manizales, del 72,7\% (Vidarte et al. 2012), se aplicó la siguiente fórmula (Montgomery \& Runger, 1996):

$$
n=\frac{\left(\sum W_{h} \sqrt{P_{h}\left(1-P_{h}\right)}\right)^{2}}{\frac{E M^{2}}{Z^{2}}}
$$

$\mathrm{n}=$ Tamaño de la muestra; $\mathrm{wh}$ : es el peso de cada estrato o la proporción de individuos de cada uno de los estratos (nh/N); $\mathrm{P}_{\mathrm{h}}$ : es la prevalencia o proporción de individuos que se estima presentan la característica a estudiar que hay en cada estrato; EM: es el margen de error (normalmente máximo el $5 \%$ ) y Z: es el nivel de confianza o confiabilidad, normalmente, la confiabilidad es del $95 \%$ y su valor de $Z$ equivale a 1,96 .

A partir de la fórmula anterior y con el objetivo de determinar el mayor nivel de precisión (5\%), con una confiabilidad del $95 \%$, se estableció el tamaño muestral para la ciudad, que fue de 451 personas; se asumió un $10 \%$ de porcentaje de pérdida, el total de la muestra fue de 529 individuos.

Posteriormente, se procedió a determinar el peso por cada grupo de edad, de manera proporcional a cada uno. Para la selección de la muestra, se empleó un diseño no probabilístico, con muestreo por cuotas (Montgomery \& Runger, 1996), donde el investigador seleccionó sujetos sobre la base de las características de la población. Fueron criterios de inclusión: que el participante se encontrara residiendo en la ciudad por un tiempo mayor a seis meses; que sea físicamente apto para la prueba; no haber consumido licor y trasnochado el 
día antes de la prueba y no haber realizado ninguna actividad vigorosa antes de la prueba

Se contó con la aceptación voluntaria para participar en el estudio y el cumplimiento de los criterios de inclusión y el diligenciamiento del consentimiento informado, aprobado por el comité de bioética de la universidad Autónoma de Manizales, según acta No. 18 de 2010. Se utilizó un instrumento con preguntas estructuradas y semiestructuradas, para obtener la información correspondiente a las variables sociodemográficas (edad, género, estrato socioeconómico, peso y talla) y de percepción sobre la actividad física (práctica de actividad física, frecuencia de práctica, tiempo de práctica) y los datos obtenidos a partir de la aplicación del test, para la clasificación del sedentarismo (Pérez et al. 2002). Este instrumento fue validado por expertos en la temática; se realizó una prueba piloto, que permitió la calibración de los evaluadores y determinó los roles a sumir, durante el proceso de recolección de información.

La sistematización de la información, se realizó el programa SPSS versión 19, licenciada por la Universidad Autónoma. Se hizo para las variables sociodemográficas un análisis univariado, a partir del cálculo de medidas de tendencia central y de dispersión, para variables cuantitativas y tablas de distribución de frecuencias, para variables cualitativas. Para establecer las correlaciones, se utilizó un análisis bivariado, con el cual, se determinó la significancia de las variables $(p<0,05)$ y para definir los niveles de asociación y dependencia, se aplicaron pruebas no paramétricas (Chi cuadrado y phi), establecidas a partir de las características propias de las variables (cualitativas). A partir del análisis bivariado, se realizó una regresión logística binaria (Gutiérrez \& De la Vara, 2003), para establecer el modelo predictivo final

\section{RESULTADOS Y DISCUSIÓN}

Con relación a las variables sociodemográficas, la muestra de estudio fue de 529 participantes, 268 hombres $(50,7 \%)$ y 261 mujeres, la media de la edad fue de 36,22 \pm 13,48 años; el 54,6\% fueron solteros al momento de la investiga- ción. Estos resultados muestran diferencia con otros estudios similares realizados en las ciudades de Manizales y de Pereira (Vidarte et al. 2012; Vélez et al. 2011).

Con relación a la descripción de las variables antropométricas, como el peso, la talla y el índice de masa corporal (IMC), se encontró que el promedio de IMC fue de $24,01 \mathrm{~kg} / \mathrm{m}^{2}+/-$ de $4,0 \mathrm{~kg} / \mathrm{m}^{2}$; el promedio del perímetro de cintura fue de $83,32 \mathrm{~cm}+/-12,921$ y el promedio del perímetro de cadera fue de $99,37 \mathrm{~cm} \mathrm{+/-} \mathrm{12,106.}$

La prevalencia del sedentarismo, para la ciudad de Medellín, calculado a partir de los resultados del presente estudio, fue de 76,2\% IC95\% (72,6\%; 78,8\%). Este dato es el resultado de cálculo de intervalos de confianza para prevalencias (Tabla 1). Este resultado es superior a lo encontrado en Manizales (Vidarte et al. 2012), donde la prevalencia de sedentarismo fue del $72,7 \%$, siendo en las mujeres el porcentaje de sedentarismo del 84\%; asimismo, el resultado parecido a lo encontrado en Pamplona (Elizondo-Armendariz et al. 2005), donde un $76,6 \%$ de las mujeres tienen un estilo de vida sedentario, mientras que en los hombres fue del $56,7 \%$. Al respecto (Elizondo-Armendariz et al. 2005) afirman en su estudio cómo el sedentarismo aumenta con la edad y evidencian cómo en el grupo de mayor edad los porcentajes de sedentarismo están por encima del 80,3\%; de igual manera, al comparar los resultados del presente estudio con el estudio realizado en Pereira (Vélez et al. 2011). Con relación a la edad, diversos estudios (Gómez et al. 2005; Irwin, 2004; Damasceno et al. 2006) muestran cómo en las edades juveniles se establece el sedentarismo y perdura a lo largo de la vida, siendo un elemento muy relevante, que sus estilos de vida están condicionados por situaciones sociales (Moscoso et al. 2013; Blasco et al. 1996).

Al comparar el género con el nivel de sedentarismo, se halló cómo en mayor porcentaje las mujeres son más sedentarias que los hombres. Al realizar las pruebas estadísticas, se evidenció que existe asociación estadísticamente significativa entre estas variables, siendo la fuerza de dependencia muy débil (Tabla 2).

Tabla 1. Distribución de la muestra niveles de sedentarismo.

\begin{tabular}{|c|c|c|}
\hline Sedentarismo & Frecuencia & Porcentaje \\
\hline SI Sedentario & 403 & $76,2 \%$ \\
\hline Sedentario severo & 145 & $27,4 \%$ \\
\hline Sedentario moderado & 258 & $48,8 \%$ \\
\hline NO Sedentario & 126 & $23,8 \%$ \\
\hline Total & 529 & $100,0 \%$ \\
\hline
\end{tabular}


Tabla 2. Comparativo entre género y sedentarismo de la muestra participante.

\begin{tabular}{|l|c|c|c|c|c|c|c|}
\hline Sedentarismo & Masculino & Femenino & Total & Chi $^{2}$ & p & Phi & p \\
\hline \multirow{2}{*}{ No sedentarios } & 89 & 37 & 126 & & & & \\
\cline { 2 - 5 } & $33,2 \%$ & $14,2 \%$ & $23,8 \%$ & & & & \\
\hline \multirow{2}{*}{ Sedentarios } & 179 & 224 & 403 & & & & \\
\cline { 2 - 5 } & $66,8 \%$ & $85,8 \%$ & $76,2 \%$ & 26,397 & $0,000 *$ & 0,223 & 0,000 \\
\hline
\end{tabular}

*p>0,05; Chi2 = asociación; Coe Phi= fuerza de asociación.

La tabla 3 muestra cómo en mayor porcentaje los que no realizan actividad física fueron sedentarios y el 68,4\% de los que sí la realizan tienen esta misma condición. Existe asociación estadísticamente significativa y una relación inversa y débil.
La tabla 4 expone cómo las personas que tienen una frecuencia de práctica de 1 y 2 veces a la semana presentan en mayor porcentaje sedentarismo. Las pruebas estadísticas aplicadas evidenciaron asociación estadísticamente significativa y una dependencia débil entre éstas variables

Tabla 3. Comparativo entre realización de actividad física y de sedentarismo de la muestra participante.

\begin{tabular}{|c|c|c|c|c|c|c|c|}
\hline Sedentarismo & $\begin{array}{c}\text { No } \\
\text { realiza }\end{array}$ & Si realiza & Total & $\mathrm{Chi}^{2}$ & p. & Phi & $\mathrm{p}$ \\
\hline \multirow{2}{*}{ No sedentarios } & 32 & 94 & 126 & & & & \\
\hline & $13,8 \%$ & $31,6 \%$ & $23,8 \%$ & & & & \\
\hline \multirow{2}{*}{ Sedentarios } & 200 & 203 & 403 & & & & \\
\hline & $86,2 \%$ & $68,4 \%$ & $76,2 \%$ & 22,889 & $0,000 *$ & 0,208 & 0,000 \\
\hline
\end{tabular}

*p>0,05; Chi2= asociación; Coe Phi= fuerza de asociación.

Tabla 4. Comparativo entre frecuencia semanal y niveles de sedentarismo de la muestra participante.

\begin{tabular}{|c|c|c|c|c|c|c|c|c|c|c|}
\hline \multirow{2}{*}{$\begin{array}{c}\text { Nivel de } \\
\text { Sedentarismo }\end{array}$} & \multicolumn{5}{|c|}{ Frecuencia Semanal de práctica } & \multirow{2}{*}{ Total } & \multirow{2}{*}{$\mathrm{Chi}^{2}$} & \multirow{2}{*}{$\mathrm{p}$} & \multirow{2}{*}{ Phi } & \multirow{2}{*}{$\mathrm{p}$} \\
\hline & $1 \mathrm{vez}$ & 2veces & 3veces & 4veces & 5veces & & & & & \\
\hline \multirow[t]{2}{*}{ No Sedentario } & 5 & 13 & 37 & 20 & 19 & 126 & \multirow{4}{*}{57,923} & \multirow{4}{*}{$0,000 *$} & \multirow{4}{*}{0,33} & \multirow{4}{*}{0,000} \\
\hline & 11,9 & 18,3 & 37,0 & 62,5 & 36,5 & 23,8 & & & & \\
\hline \multirow{2}{*}{ Sedentarios } & 37 & 58 & 63 & 12 & 33 & 403 & & & & \\
\hline & 88,1 & 81,7 & 63,0 & 37,5 & 63,5 & 76,2 & & & & \\
\hline
\end{tabular}

*p>0,05; Chi2 = asociación; Coe Phi= fuerza de asociación.

Las variables que presentaron asociación estadísticamente significativa con el sedentarismo y que hacen parte del análisis logístico final son: género, IMC, consumo de café, tipo de práctica, frecuencia semanal y tiempo de práctica. Se excluyen del modelo las variables tiempo, frecuencia y tipo de práctica, por considerarse variables endógenas (es decir, son inherentes a la práctica deportiva); el resto de variables no fueron estadísticamente significativas (Tabla 5).

En este estudio, se estableció asociación entre el sedentarismo y el IMC, donde el $72,2 \%$ de los que presentaron un índice de masa corporal (IMC) normal son sedentarios y el 
$83,7 \%$ de los de IMC sobrepeso son sedentarios, datos muy similares a los encontrados en otros estudios sobre obesidad (Elizondo-Armendariz et al. 2005; Montenegro \& Rubiano, 2006; Vélez et al. 2011; Cabral et al. 2003; Blasco et al. 1996).
Se seleccionaron diferentes combinaciones entre las variables analizadas o posibles predictoras, con el fin de obtener el logaritmo matemático que prediga el sedentarismo en la población objeto de estudio. El modelo explorado tuvo en consideración la significancia individual de los Beta y las pruebas

Tabla 5. Resumen de asociaciones bivariadas con los niveles de sedentarismo.

\begin{tabular}{|l|c|c|c|c|}
\hline & $\begin{array}{c}\text { Chi-cuadrado } \\
\text { de Person }\end{array}$ & $\mathbf{p}$ & Phi & p \\
\hline Edad & 2,288 & 0,130 & 0,066 & 0,130 \\
\hline Género & $\mathbf{2 6 , 3 9 7}$ & $\mathbf{0 , 0 0 0}$ & $\mathbf{0 , 2 2 3}$ & $\mathbf{0 , 0 0 0}$ \\
\hline IMC & $\mathbf{8 , 7 7 9}$ & $\mathbf{0 , 0 0 3}$ & $\mathbf{0 , 1 2 9}$ & $\mathbf{0 , 0 0 3}$ \\
\hline Estado civil & 0,002 & 0,965 & 0,002 & 0,965 \\
\hline Tipo de práctica & $\mathbf{2 2 , 8 8 9}$ & $\mathbf{0 , 0 0 0}$ & $-\mathbf{0 , 2 0 8}$ & $\mathbf{0 , 0 0 0}$ \\
\hline Frecuencia semanal & $\mathbf{5 7 , 9 2 3}$ & $\mathbf{0 , 0 0 0}$ & $\mathbf{0 , 3 3 1}$ & $\mathbf{0 , 0 0 0}$ \\
\hline Tiempo de práctica & $\mathbf{3 4 , 3 9 6}$ & $\mathbf{0 , 0 0 0}$ & $\mathbf{0 , 2 5 5}$ & $\mathbf{0 , 0 0 0}$ \\
\hline Consumo de tabaco & 0,028 & 0,868 & $-0,007$ & 0,868 \\
\hline Consumo de alcohol & 0,013 & 0,908 & 0,005 & 0,908 \\
\hline Consumo de cafeína & $\mathbf{5 , 4 1 0}$ & $\mathbf{0 , 2 0}$ & $-\mathbf{0 , 1 0 1}$ & $\mathbf{0 , 0 2 0}$ \\
\hline
\end{tabular}

*p>0,05; Chi2 = asociación; Coe Phi $=$ fuerza de asociación

de bondad de ajuste del modelo (Anderson et al. 2000). La ecuación o función logística del modelo estimado fue:

$$
p(\text { Sedentarismo }=1)=\frac{1}{1+e^{-\left(-0,772+1,207^{*} \text { género }+0,755 * I M C-0,524 * \text { Consumo cafe }\right)}}
$$

Donde $\mathrm{p}_{\mathrm{i}}$ es la probabilidad de una persona ser sedentaria.

En la tabla 6, se establece el nivel de significancia de las variables descritas género, índice de masa corporal y consumo de cafeína; siendo estas estadísticamente significativas y representativas para el modelo de regresión global, empleado para la ciudad de Medellín. Dicho modelo al validarse, a través del test de Wald, muestra un coeficiente significativo.

Al comparar los resultados del estudio de Medellín con otros modelos de regresión logística multivariados, destacan la importancia de la obesidad en todas las patologías estudiadas, con riesgos casi tres veces mayor para diabetes, ajustando por otras variables de confusión (Gómez-Cabello et al. 2012; Ruiz et al. 2012; Martínez et al. 2008). En Chile, Serón et al. (2010) y Ratner et al. (2008) encontraron que la frecuencia entre los niveles de actividad física fue significativamente diferente entre los hombres y las mujeres; los resultados de estos estudios muestran valores de niveles de actividad física bajos o moderados, especialmente, en las mujeres. Dato contrario en otros estudios, donde el sobrepeso es menor en las mujeres (Damasceno et al. 2006; Rodríguez et al. 2013).

En relación a la percepción de práctica de actividad física, frecuencia y tiempo de práctica (Martínez et al. 2008; Weiss et al. 2007), hicieron un análisis con la evaluación de los conocimientos, actitudes y prácticas de actividad física y sus factores asociados en personas de ambos sexos y diferentes grupos de edad y encontraron cómo el $21,2 \%$ realiza suficiente actividad física, para cuidar su salud.

Chuliá et al. (2005) analizaron la frecuencia de actividad física con la clase social, preocupándose por los cambios en los estilos de vida y hábitos, como fumar, consumir alcohol y la experiencia con drogas, como la marihuana. En la ciudad de Medellín, se encontró que el nivel de sedentarismo y los hábitos de vida, como fumar, beber y consumir sustancias psicoactivas, no se asocian (Elizondo-Armendariz et al. 2005; Ruiz-Risueño, 2010). Al analizar la misma relación, estos autores encontraron que no existe asociación entre el consumo de tabaco y tener un estilo de vida sedentario, aunque el porcentaje de hombres sedentarios sea algo superior entre los fumadores.

El consumo de café fue estadísticamente significativo y se convirtió en la variable predictora del sedentarismo en Me- 
Tabla 6. Variables de la ecuación del modelo predictivo.

Variables en la ecuación

\begin{tabular}{|l|l|r|r|r|r|r|r|}
\hline \multicolumn{2}{|c|}{ Variables } & \multicolumn{1}{c|}{ B } & E.T. & \multicolumn{1}{c|}{ Wald } & gl & \multicolumn{1}{c|}{ p } & \multicolumn{1}{|c|}{ Exp(B) } \\
\hline \multirow{2}{*}{ Género(1) } & 1,207 &, 225 & 28,678 & 1 & $0,000 *$ & 3,345 \\
\cline { 2 - 8 } & IMC2(1) &, 755 &, 242 & 9,704 & 1 & $0,002 *$ & 2,127 \\
\cline { 2 - 8 } & Cafeína(1) &,- 524 &, 233 & 5,047 & 1 & $0,025 *$ &, 592 \\
\cline { 2 - 8 } & Constante &, 772 &, 221 & 12,176 & 1 & $0,000 *$ & 2,163 \\
\hline
\end{tabular}

Variable(s) introducida(s) en el paso 1: Género, IMC2, Cafeína. B= Beta; Wald= coeficientes; $* \mathrm{p}>0,05 ; \mathrm{Ex}(\mathrm{B})=$ o razón de probabilidad

dellín, a partir del modelamiento realizado. El consumir café, se asocia con los factores de riesgo relacionados con estilos de vida poco saludables, sus elementos logran efectos neurofisiológicos que estimulan el sistema nervioso y mejoran el estado de alerta de la persona y se produce un aumento de la termogénesis y del gasto energético, lo cual, es un factor beneficioso a largo plazo, en el caso de personas con obesidad (Gotteland, 2007).

Otro estudio, concluye que existe una relación entre la dosis y la respuesta entre consumo de café y niveles de colesterol y triglicéridos (Socarrás \& Bollet, 2010). Con relación a los hábitos de vida relacionados con el sedentarismo, en España (Martínez-Ros et al. 2003) concluyeron que los únicos factores que tienen asociación en varones son el consumo de tabaco, donde los fumadores realizan menos actividad física, que los que no fuman.

Al comparar las variables de mayor peso estadístico en el modelamiento del presente estudio, es decir, predictoras del sedentarismo (el género, el consumo de cafeína y el índice de masa corporal), se encontró concordancia en las variables género y de IMC, de los estudios desarrollados por Reig et al. (2003); Salazar et al. (2013); Bernstein et al. (1999); Godard et al. (2008).

Se hace necesario insistir en estrategias que mejoren la práctica de actividad física en la población; lo anterior debe ser complementado con la generación de procesos y de estrategias educativas y de monitoreo frente al comportamiento sedentario, donde se estimule, permanentemente, la práctica constante de la actividad física, como política de la promoción de la salud. Por tanto, las políticas gubernamentales deben orientar los recursos hacia la promoción de hábitos saludables.

Conflictos de interés: El manuscrito fue preparado y revisado con la participación de todos los autores, quienes declara- mos que no existe ningún conflicto de intereses que ponga en riesgo la validez de los resultados presentados.

\section{BIBLIOGRAFÍA}

1. ALEMÁN, C.; SALAZAR, W. 2004. Nivel de actividad física, sedentarismo y variables antropométricas en funcionarios públicos. Universidad de Costa Rica. Rev. Cienc. Ejerc. Salud. 4(1):1-12.

2. ANDERSON, R.; TATHAM, R.L. (AUTHOR), HAIR Jr., J.F. 2000. Análisis Multivariante de Datos. $5^{\mathrm{a}}$ Ed. Prentice Hall. 832p.

3. BERNSTEIN, S.M.; MORABIA, A.; SLOUTSKIS, D. 1999. Definition and prevalence of sedentarism on an urban population. Am. J. Public Health. 89:862-27.

4. BLASCO, T.; CAPDEVILA, L.; PINTANEL, M.; VALIENTE, M.; CRUZ, J. 1996. Evolución de los patrones de actividad física en estudiantes universitarios. Rev. Psicol. Deporte. 9-10:51-63.

5. BOUCHARD, C.; SHEPARD, R.J.; STEPHENS, T. 1994. Physical activity, Fitness and Health. Int. Proc. Consensus Statement. Champaing: Human Kinetics. Publishers Inc. p.569-915.

6. CABRAL, P.C.; MELO, C.A.; AMADO, C.F. 2003. Evaluación antropométrica y dietética de hipertensos atendidos en ambulatorio de un hospital universitario. Rev. Nutr. Campinas. 16(1):61-71.

7. CAPDEVILA, L.; PINTANEL, M.; VALERO, M.; OCAÑA, M.; PARRADO, E. 2006. Estrategias de intervención para promocionar la actividad deportiva en la población universitaria femenina. Madrid: Consejo Superior de Deportes. Serie ICd no 46 ed. p.84-95. 
8. CHULIÁ, M.; FERRER, E.; LIZAMA, N.; MARTIN, S.; MONRABAL, C. 2005. El sedentarismo en los jóvenes universitarios. Educare 21. Rev. Electr. Formación Enfermera. Disponible desde Internet en: http://www.enfermeria21.com/educare/Generalitats/componentes/articulos/enlace_articulo. php?Mg $==\& \& M j \mathrm{c}=\& M T M w N Q=\quad\left(c^{-}\right.$acceso 03/02/2014).

9. DAMASCENO, M.; SILVA, L.; ALMEIDA, V.; ATAÍDE, M.; SILVA, A. MACEDO, S. 2006. Obesidad y exceso de peso: identificación de casos entre los trabajadores del área de salud. Enf. Integral. 3:15-21.

10. ELIZONDO-ARMENDARIZ, J.J.; GRIMA, F.; AGUINAGA, I. 2005. Prevalencia de la actividad Física y su relación con variables sociodemográficas y estilo de vida en la población de 18 a 65 años de Pamplona. Rev. Esp. Salud Pública. 79:559-567.

11. GARCÍA, L.V.; CORREA, J.E. 2007. Muévase contra el sedentarismo. Universidad ciencia y desarrollo. Programa de divulgación científica. Tomo II, fascículo 7. Universidad del Rosario. Disponible en: www. Urosario.edu.co/investigación/tomo2/fasciculo7/index. html (con acceso 25/02/2014).

12. GODARD, M.C.; RODRÍGUEZ, M.P.; DÍAZ, N.; LERA, L.; SALAZAR, R.G.; BURROWS, A.R. 2008. Valor de un test clínico para evaluar actividad física en niños. Rev. Méd. Chile. 136:1155-1162.

13. GÓMEZ-CABELLO, A.; VICENTE-RODRÍGUEZ, G.; PINDADO, M.; VILA, S.; CASAJÚS, J.A.; PRADAS DE LA FUENTE, F.; ARA, I. 2012. Mayor riesgo de obesidad y obesidad central en mujeres post-menopáusicas sedentarias. Nutr. Hosp. 27(3):865-870.

14. GÓMEZ, L.F.; DUPERLY, J.; LUCUMI, D.I. 2005. Physical activity levels in adults living in Bogotá (Colombia): prevalence and associated factors. Gac. Sanit. 19(3):206-213.

15. GOTTELAND, M.; DE PABLO, S. 2007. Algunas verdades sobre el café. Rev Chil Nutr 34(2):105-115.

16. GUTIÉRREZ, H.; DE LA VARA R. 2003 Análisis y Diseño de Experimentos, McGraw-Hill. p.234.

17. IRWIN, J.D. 2004. Prevalence of university students' sufficient physical activity: A systematic review. Perceptual and Motor Skills. 98(3):927-943.
18. MARTÍNEZ, E.; SALDARRIAGA, J.F.; SEPÚLVEDA, F.E. 2008. Actividad física en Medellín: desafío para la promoción de la salud. Rev. Fac. Nac. Salud Públ. 26(2):117-123.

19. MARTÍNEZ-ROS, M.T.; TORMO, M.J.; PÉREZ-FLORES, D.; NAVARRO, C. 2003. Actividad física deportiva en una muestra representativa de la población de la Región de Murcia. Gac. Sanit. 17:11-99.

20. McGINNIS, J.M.; FOEGE, W.H. 1993. Actual causes of death in the United States. JAMA. 270(18):22072212.

21. MONTENEGRO, Y.M.; RUBIANO, O.F 2006. Sedentarismo en Bogotá, Características de una sociedad en riesgo. Umbral Cient. 9:33-45.

22. MONTGOMERY, C.D.; RUNGER, C.G. 1996. Probabilidad y Estadística aplicadas a la ingeniería. Editorial McGraw- Hill. 198p.

23. MOSCOSO, D.; MARTÍN, D.; PEDRAJAS, N.; SÁNCHEZ, R. 2013. Sedentarismo activo, ocio, actividad física y estilos de vida de la población española. Rev Fed. Esp. Med. Deporte Conf. Iberoam. Med. Deporte. 158:341-347.

24. ORGANIZACIÓN MUNDIAL DE LA SALUD -OMS-. 2002. Informe sobre la salud en el mundo: Reducir los riesgos y promover una vida sana. 65p.

25. ORGANIZACIÓN MUNDIAL DE LA SALUD -OMS-. 2009. Global health risks: Mortality and burden of disease attributable to selected major risks. Geneva: World Health Organization. 70p.

26. ORGANIZACIÓN PANAMERICANA DE LA SALUD -OPS. 2005. Salud en las Américas. Disponible desde Internet en http://www.per.opsoms.org/sea-07/SEA2007-3.pdf (con acceso 20/01/14).

27. PATE, R.R.; O’NEIL, J.R.; LOBELO, F. 2008. The evolving definition of "sedentary". Exerc. Sport Sci. Rev. 36:73-78.

28. PÉREZ, A.; SUÁREZ, R.; GARCÍA, G.; ESPINOSA, A.; LINARES, D. 2002. Propuesta de variante del test de sedentarismo y su validación estadística. La Habana, Cuba: Facultad de Cultura Física, Universidad de Cienfuegos. Disponible desde Internet en: www.fac. org.ar/1/.../Sedentarismo\%20Cuba.htm (con acceso 06/02/14). 
29. RAMÍREZ, W.; VICACCIA, S.; SUÁREZ, G.R. 2004. impacto de la actividad física y el deporte sobre la salud, la cognición, la socialización y el rendimiento académico: una revisión teórica. Rev. Estud. Soc. 18:67-75.

30. RATNER, R.; SABAL, J.; HERNÁNDEZ, P.; ROMERO, D.; ATALAH, D. 2008. Estilos de vida y estado nutricional de trabajadores en empresas públicas y privadas de dos regiones de Chile. Rev. Méd. Chile. 136:14061414.

31. REIG, A.; CABRERO, J.; FERRER, R.; RICHART, M. 2003. La calidad de vida y el estado de salud de los estudiantes universitarios. Alicante. Biblioteca Virtual Universal. Universidad de Alicante. 66p.

32. RODRÍGUEZ, F.; PALMA, X.; ROMO, A.; ESCOBAR, D.; ARAGU, B.; ESPINOZA, L.; McMILLAN, M.; GALVEZ, J. 2013. Hábitos alimentarios, actividad física y nivel socioeconómico en estudiantes universitarios de Chile. Nutr. Hosp. 28(2):447-455

33. RUIZ, G.; DE VICENTE, E.; VEGARA, J. 2012. Comportamiento sedentario y niveles de actividad física en una muestra de estudiantes y trabajadores universitarios. J. Sport Health Res. 4(1):83-92.

34. RUIZ-RISUEÑO, J. 2010. Hábitos deportivos y estilos de vida de los escolares de ESO en los municipios de la provincia de Almería. Retos. Nuevas Tendencias Ed. Fís., Dep. Recr. 17:49-53.

35. SALAZAR, C.; FEU, S.; VIZUETE, M.; DE LA CRUZ- SÁNCHEZ, E. 2013. IMC y actividad física de los estudiantes de la universidad de Colima. Rev Int. Med. Cienc. Act. Fís.l Dep. 13(51):569-610.
36. SECRETARÍA DE TURISMO Argentina en movimiento. 2000. Hábitos deportivos de la población argentina, con soporte calificado del Instituto Nacional de Estadística y Censos (INDEC). p.1-143.

37. SERÓN, P.; MUÑOZ, S.; LANAS, F. 2010. Nivel de actividad física medida a través del cuestionario internacional de actividad física en población chilena. Rev. Med. Chile. 138:1232-1239.

38. SOCARRÁS, M.M.; BOLET ASTOVIZA, M. 2010 Alimentación saludable y nutrición en las enfermedades cardiovasculares. Rev Cub. Invest. Bioméd. 29(3):353-363.

39. VARELA, M.T.; DUARTE, C.; SALAZAR, I.C.; LEMA, L.F.; TAMAYO, J.A. 2011. Actividad física y sedentarismo en jóvenes universitarios de Colombia: prácticas, motivos y recursos para realizarlas. Col. Med. 42:269-277.

40. VÉLEZ, C.; VIDARTE, J.A.; RÍOS, D.M.; MUÑOZ, A.P. 2011. Prevalencia de actividad física y factores relacionados en la población de 18-60 años - Pereira 2011. Rev. Méd. Risaralda. 17(2):85-90.

41. VIDARTE, J.A.; VÉLEZ, C.; PARRA, J. 2012. Niveles de sedentarismo en población de 18 a 60 años. Manizales, Colombia. Rev. Fac. Nac. Salud Públ. 14(3):415-426.

42. WEISS, D.R.; O'LOUGHLIN, J.L.; PLATT, R.W.; PARADIS, G. 2007. Five-year Predictors of physical activity decline among adults in low-income communities: a prospective study. Int J. Behav. Nutr. Phys. Act. 4:2.

Recibido: Enero 27 de 2014

Aceptado: Abril 22 de 2014

Como citar:

Vidarte, J.A.; Vélez, C.; Iglesias, L.E.; Galvis, Y. 2014. Variables predictoras de los niveles de sedentarismo en población de 18 a 60 años, Medellin 2012. Rev. U.D.C.A Act. \& Div. Cient. 17(1): 65-72. 